\section{e0429 PATIENTS WITH TYPE 2 DIABETES EXHIBIT IMPAIRED MOBILISATION OF ENDOTHELIAL PROGENITOR CELLS AFTER ACUTE MYOCARDIAL INFARCTION MEDIATED BY VEGF/AKT/ENOS/MMP9 PATHWAY}

doi:10.1136/hrt.2010.208967.429

Shen Yu, Lu Wen, Gu Rong, Wan Kun, Kang Lina. 'Department of Cardiology, Nanjing Gulou Hosital

Background Type 2 diabetes mellitus (T2DM) is a main risk of coronary artery disease (CAD), with impairment of endothelial progenitor cells (EPCs) function thought to be a contributing factor. EPCs from bone marrow are mobilised in response to ischaemia, and play an important role in neovasculation.

Objective The aim of this study is to investigate EPC mobilisation after acute myocardial infarction (AMI) in T2DM patients is responsible for the impairment of neovasculation. We hypothesised that it is mediated by the downregulation of vascular endothelial growth factor (VEGF) pathway in bone marrow.

Research design and methods We recruited 22 patients who were admitted to hospital following AMI. 11 had T2DM and 11 were non-diabetic. Circulating CD $45^{-/ \text {low }} / \mathrm{CD} 34^{+} / \mathrm{CD} 133^{+} / \mathrm{KDR}^{+} \mathrm{EPCs}$ were quantified by flow cytometry, at different time points up to Day 28 post-AMI, as well as plasma vascular endothelial growth factor (VEGF), stromal cell-derived factor (SDF) $-1 \alpha$ and high sensitivity C-reactive protein (hsCRP). Rats with fat-diet, streptozotocin-induced T2DM were used to test the EPC-mobilising pathway in bone marrow. Circulating EPCs were identified as

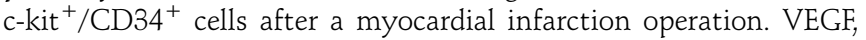
phosphorylation of Akt, endothelial nitric oxide synthase (eNOS), and activity of matrix metalloproteinase (MMP)-9 in bone marrow were measured.

Results Following AMI, patients with T2DM exhibited a delay (peak time: Day 7 vs Day 5) and a decrease in magnitude (peak level: $140 \pm 48 / 10^{6}$ mononuclear cells (MNCs) vs $246 \pm 100 / 10^{6} \mathrm{MNCs}$, $\mathrm{p}<0.05)$ of EPC mobilisation, as compared to non-diabetic patients. This is similar to what we found in the T2DM rat model following AMI surgery. The first peak of EPC mobilisation in diabetic rats occurred 2 days later (Day 3 vs Day 1), and lower than nondiabetic rats $\left(101 \pm 44 / 10^{5} \mathrm{MNCs}\right.$ vs $260 \pm 64 / 10^{5} \mathrm{MNCs}$ respectively, $\left.\mathrm{p}<0.05\right)$. In the human study, the impairment in EPC mobilisation was accompanied by augmented upregulation of plasma VEGF, stromal cell-derived factor- $1 \alpha$ and high sensitivity C-reactive protein following AMI, in T2DM vs non-diabetic patients. After myocardial infarction, downregulation of VEGF, Akt, eNOS and MMP9 in bone marrow were detected in T2DM rats comparing to non-diabetic group.

Conclusions This is the first demonstration that bone marrowderived EPC mobilisation following AMI is impaired in patients with T2DM. Such impairment is likely to have important contribution to the poor collateralization observed in such patients in response to vascular occlusive disease.

\section{e0430 HIGHSENSITIVITY CREACTIVE PROTEIN LEVELS AT ADMISSION CAN NOT BE USED TO PREDICT INHOSPITAL MORTALITY IN PATIENTS WITH ACUTE MYOCARDIAL INFARCTION A RETROSPECTIVE STUDY}

doi:10.1136/hrt.2010.208967.430

Chen Ying, Chen Hong. Department of Cardiology Peking University People's Hospital

Objective To investigate the possible influencing factors of serum high-sensitivity C-reactive protein (hs-CRP) levels on admission, and to assess the the association between admission serum hs-CRP levels and mortality in patients admitted because of acute myocardial infarction (AMI).
Methods A total of 609 patients with AMI from Peking University People's Hospital between January, 2005 and December, 2009 were enrolled in this retrospective analysis. The clinical data including age, gender, traditional risk factors of atherosclerosis, history of cardiovascular disease, laboratory test results including hs-CRP, lipid, serum creatinine, myocardial enzymes, and echocardiographic results were collected. The potential influencing factors of hs-CRP levels, and correlations between admission hs-CRP and in-hospital outcomes were statistically analysed by the use of logistic regression.

Results The median serum hs-CRP level of 609 patients with AMI is $8.22 \mathrm{mg} / 1$ (3.40 mg/l, $12.99 \mathrm{mg} / \mathrm{l})$. Patients with hs-CRP level>3.0 $\mathrm{mg} / \mathrm{l}$ accounted for $78.1 \%$. Hs-CRP correlated positively with the time interval from onset of pain until the hs-CRP levels were tested and myocardial enzymes and negatively with statin use before admission. With increasing hs-CRP levels $(<1.0,1.0$ to $3.0,>3.0$ to 10.0 and $>10.0 \mathrm{mg} / \mathrm{l}$ ) left ventricular ejection fraction decreased, while incidence rate of aneurysm formation, ventricular septal perforation, mitral valve prolapse and apical thrombus was no significant difference. A total of $22(3.6 \%)$ patients died during hospitalisation, of which 21 (3.4\%) for cardiac death. Increased hsCRP levels were associated with increased risk of in-hospital death $(\mathrm{OR}=1.134,95 \% \mathrm{CI}, 1.046$ to $1.228, \mathrm{p}=0.002)$. When controlling for the confounding effect of age, gender, traditional risk factors of atherosclerosis, history of cardiovascular disease, cardiac function and cardiac enzymes, patients with hs-CRP $\geq 11 \mathrm{mg} / 1$ had a 3 -fold increased risk of death compared with those hs-CRP $<11 \mathrm{mg} / \mathrm{L}$ $(\mathrm{OR}=4.009,95 \% \mathrm{CI}, 1.175$ to $13.673, \mathrm{p}=0.027)$. After further adjusted for renal function, correlation between elevated hs-CRP and death reduced below the significant level $(\mathrm{OR}=2.519,95 \% \mathrm{CI}$, 0.673 to $9.433, \mathrm{p}=0.170$ ).

Conclusions There was a weak association between admission serum hs-CRP levels and in-hospital mortality in patients with AMI. The prognostic value of hs-CRP levels at admission was limited.

\section{e0431 INCREASED EXPRESSION OF INTEGRIN-LINKED KINASE IMPROVES CARDIAC FUNCTION AFTER MYOCARDIAL INFARCTION IN THE SWINE}

doi:10.1136/hrt.2010.208967.431

Wen Lu, Wen Lu, Biao Xu.

Objective and backgroud Left Cardiac remodelling is generally accepted as a determinant of the clinical course of heartfailure (HF). After myocardial infarction, cardiomyocyte loss and increased load trigger genome expression resulting in molecular, cellular and interstitial changes and manifested clinically as changes in size, shape and function of the heart. Prevention or attenuation of these signalling processes is an important goal of anti-remodelling therapy. Integrin-linked kinase (ILK), a widely expressed in mammalian tissues serine/threonine protein kinase, plays an important role in transducing cell-matrix interaction-induced biomechanical signals which regulate cytoskeletal remodelling, angiogenesis, cell growth, proliferation, survival and differentiation. Recently, ILK has been reported to be an critical component of the cardiac stretch sensor which regulates cardiac contractility, compensatory hypertrophy, survival and repair. In the previously study, we have found ILK could attenuate LV remodelling and improve the heart function after myocardial infarction in rats. In the present study, we investigated whether ILKcould act a same role after acute myocardial infarction in swine.

Methods Recombinant adenoviral vector containing both human wild-type ILK and humanised recombinant green fluorescent protein 